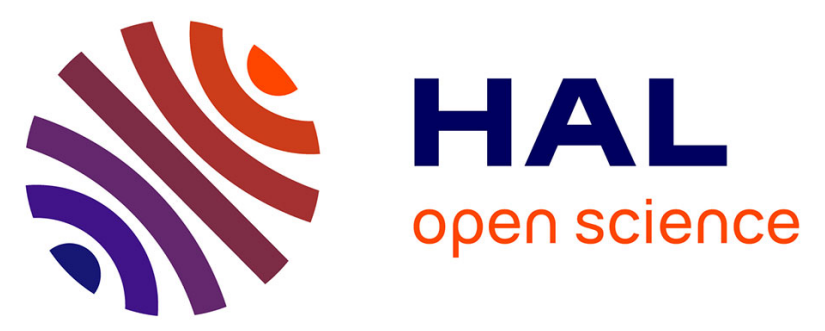

\title{
The use of impulse and stepped frequency radar to characterize the hydric behaviour of a porous pavement structure
}

\author{
Xavier Derobert, A. Ihamouten, David Guilbert, Frédéric Bosc, Frédéric
}

Bernardin

\section{To cite this version:}

Xavier Derobert, A. Thamouten, David Guilbert, Frédéric Bosc, Frédéric Bernardin. The use of impulse and stepped frequency radar to characterize the hydric behaviour of a porous pavement structure. Near Surface Geophysics, 2019, 17 (3), pp.201-212. 10.1002/nsg.12044 . hal-02133940

\section{HAL Id: hal-02133940 \\ https://hal.science/hal-02133940}

Submitted on 9 Jun 2021

HAL is a multi-disciplinary open access archive for the deposit and dissemination of scientific research documents, whether they are published or not. The documents may come from teaching and research institutions in France or abroad, or from public or private research centers.
L'archive ouverte pluridisciplinaire HAL, est destinée au dépôt et à la diffusion de documents scientifiques de niveau recherche, publiés ou non, émanant des établissements d'enseignement et de recherche français ou étrangers, des laboratoires publics ou privés. 


\title{
The use of impulse and stepped-frequency radar to characterize the hydric behaviour of a porous pavement structure
}

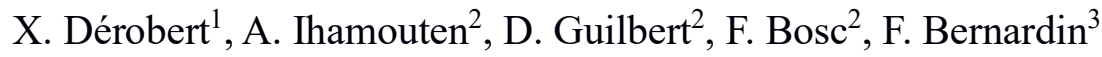 \\ ${ }^{1}$ IFSTTAR, CS5004, 44344 Bouguenais, France \\ ${ }^{2}$ CEREMA, DTer Ouest, DLRCA, 49136, Les Ponts-de-Cé, France \\ ${ }^{3}$ CEREMA, DTer Centre-Est, 63100, Clermont-Ferrand, France
}

\section{ABSTRACT}

This study focuses on examining the behaviour of an innovative asphalt pavement which is created as a solar energy collector, using non-destructive testing involving ground-penetrating radar. The concept of heat exchanger is based on the use of drainage asphalt in the bonding layer through which a heated fluid flows via gravity to de-ice the roadway. In order to develop hydrothermal models for a test site representing such a pavement, the saturated porous layer assumption was required when the water level in each tank (up- and down-stream of the structure) was on top of the porous layer. Two GPR techniques were tested at this test site: a ground-coupled impulse radar and an air-coupled steppedfrequency radar. The impulse GPR, a high-efficiency non-destructive testing technique which is widely used in civil engineering, provides accurate geometric information, especially for pavement investigation. In the second innovative approach, air-coupled stepped-frequency radar was combined with full waveform inversion to obtain quantitative information, while retrieving the electromagnetic properties of the successive pavement layers.

We concentrated on the early-stage water imbibition in the pavement structure, using the impulse GPR to estimate the fluid transfer velocity and both GPR techniques to verify the saturated porous layer assumption in the steady state. Ground-coupled radar could follow the water front and could capture different water-transfer behaviours in the porous asphalt layer. Our observation could be explained by 

similar results to ground-coupled GPR, but could quantitatively estimate also the changes within the porous layer during the test.

In the context of climate change and road designs promoting economical and environmentally

33 friendly maintenance, innovative anti-ice road structures are necessary. The principle is to use an

34 asphalt pavement as an energy source, in order to prevent the formation of ice and snow on roads by

35 storing the solar energy captured on the road during hot periods and using this captured energy during

36 cold periods. Successful, economical and environment-friendly anti-icing techniques are needed to

37 increase road safety and mobility.

38 Various innovations for using asphalt pavements as an energy source have been studied earlier (e.g.,

39 Pan et al. 2015; Andriopoulos 2012), the most recent ones focusing on asphalt solar collectors (Bobes et al. 2013). The present study focuses on another concept of heat exchange installations based on the use

41 of drainage asphalt in the bonding layer through which a heated fluid flows via gravity to de-ice the 42 roadway (Asfour et al. 2016). A full-scale experiment site was designed to test the various technologies,

43 from the supply of heat transfer fluid and the heat-transfer behaviour through the pavement structure, to

44 geothermal storage.

45 To better fit the modelling of thermal fluid flows to our experiment, non-destructive testing (NDT)

46 was performed in this test site using two ground-penetrating radar (GPR) techniques. GPR is a high-

47 efficiency NDT technique which is widely used in civil engineering (e.g., Lai et al. 2017). GPR 
provides accurate geometric information, especially for pavement investigation (e.g., Saarenketo and

49 Scullion 2000; Loizos and Plati 2007).

50 This study concentrated on water imbibition at the early stage in a pavement structure.

51 Investigations were carried out using an impulse GPR with ground-coupled antennas to estimate the

52 fluid transfer velocity. Another complementary NDT was performed using static air-coupled stepped-

53 frequency radar (SFR) combined with full waveform inversion (FWI) to quantitatively estimate the

54 changes of the saturated trench in the porous layer, during the test. This innovative approach allows

55 very-high frequency investigations, offering high resolution (Dérobert et al. 2001) in combination with

56 calibrated wave propagation, which can be fitted with the results of an accurate forward model

57 describing the complete signal. This methodology enables retrieving the electromagnetic (EM)

58 properties of the successive pavement layers (Guan et al. 2017).

59 In the following, after a discussion on the test site, the NDT techniques and the monitoring

60 methodology will be presented. These will be followed by results and discussion.

61

POROUS PAVEMENT TEST SITE

A pavement structure was built in Egletons (France) in order to experimentally validate the heat

64 exchanger function to retrieve solar energy in summer and defreeze road surfaces in winter (Fig. 1a).

65 The pavement structure is composed of three layers (Fig. 1b): a wearing course of semi-phaneritic 66 asphalt concrete $6 \mathrm{~cm}$ thick (WL); a bonding course of $0 / 14$ porous asphalt $8 \mathrm{~cm}$ thick (PAL); and a

67 base layer of asphalt concrete $5 \mathrm{~cm}$ thick (BL). The porous asphalt layer has a porosity of $20 \%$, through

68 which a fluid (water) circulates via gravitational flow because of the slope (2\%). The dimensions of this

69 structure are about $3.2 \mathrm{~m}$ in cross section and less than $4 \mathrm{~m}$ in longitudinal section (traffic lane). Lateral

70 borders are made watertight to cause the water to flow in a single direction. 
a)

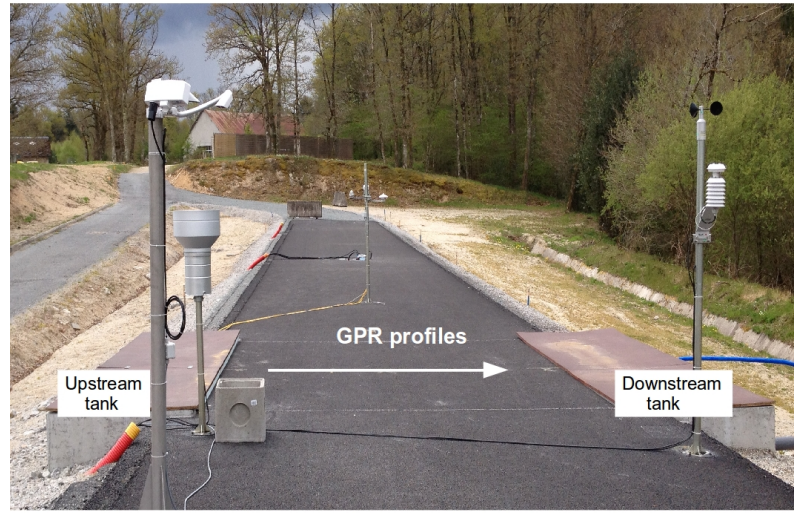

b)

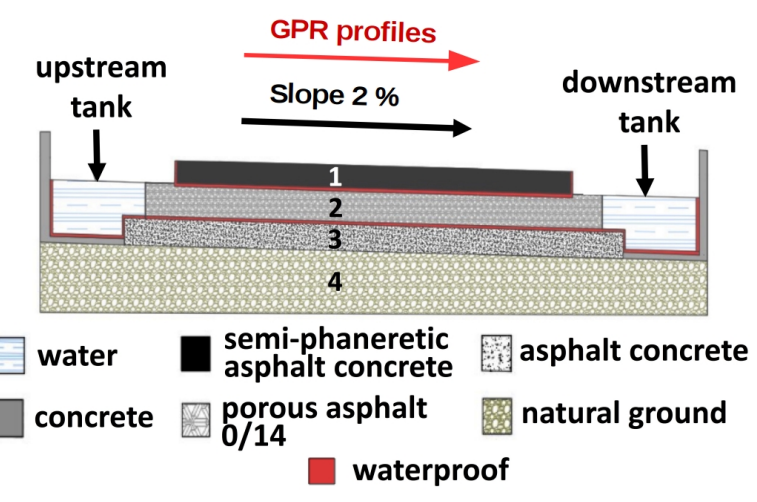

71 Fig. 1. Diagram showing the porous pavement test site and the orientation of the transverse GPR profiles

Two tanks, one upstream and another downstream, were built to supply and recover circulating fluid in the asphalt drainage layer. Fluid circulation was maintained by a watertight seal between the porous asphalt course and the underlying layer. A pump was used to control different hydraulic head losses between the upstream and downstream tanks (fluid levels in the tanks). Different hydraulic regimes were achieved with varying pump powers. In order to develop hydrothermal models for such a pavement, we need to verify the saturated porous layer assumption when the water level in each tank is on top of the porous layer.

\section{NON-DESTRUCTIVE MONITORING TECHNIQUES}

\section{Impulse radar technique}

Two commercial, impulse GPR systems (SIR3000 by GSSI) were used in parallel with groundcoupled $2.6 \mathrm{GHz}$ antennas to limit the duration of the measurement series. The setting parameters were: time range of $10 \mathrm{~ns}, 512$ sampling for A-scans, frequency-band filtering $500 \mathrm{MHz}-5 \mathrm{GHz}$, constant 
time gain $-6 \mathrm{~dB}$, and a spatial sampling of $1 \mathrm{~cm}$. At center frequency of $2.6 \mathrm{GHz}$, the first two interfaces, between the wearing layer and the porous asphalt layer (WL/PAL) and between the porous asphalt layer and the base layer (PAL/BL), could be resolved before water inflow, at about 1 and $2.2 \mathrm{~ns}$ respectively, due to high EM contrasts.

In order to accurately monitor water inflow and transfer through the porous asphalt layer, 9 parallel lines in the direction of the gravity slope, with a distance of $0.5 \mathrm{~m}$ between the lines, were periodically surveyed (transverse GPR profiles mentioned in Fig.1b). Every 7 minutes, one impulse GPR system surveyed from Line 0 to Line 4, while the second impulse GPR system did the same from Line 8 to Line 5. This sequence lasted about 3 minutes. After an initial series (series 0 or time T0) of acquisition, water circulation was started from the upstream tank inside the porous asphalt layer. The saturated steady state was considered established after 1.5 hour, leading to 18 series of radar measurements in 115 minutes.

After two hours of a permanent saturated state maintained by a pump controlling the water flow inside the pavement, the supply was stopped allowing the natural leakage of water inside the porous asphalt layer to flow to the downstream tank. This second experiment led to 16 series of impulse GPR measurements in 130 minutes (about every 8 minutes).

The objective was to pick the double travel times of radar echoes from the different interfaces, mainly the water front in the porous layer during the imbibition processes, and to monitor the fluid flow and verify whether the water level joins the top of the PAL or whether some air lenses remain.

\section{SFR technique}

The second NDT method consisted of a homemade stepped-frequency radar (SFR) system with an ultra-wide band (UWB), air-coupled antenna $(0.8-8 \mathrm{GHz})$ used in near-field conditions (at $4 \mathrm{~cm}$ from the the surface of the pavement structure). For this experiment, SFR measurements were performed in a 
static mode to ensure an accurate height during the monitoring, between lines 6 and 7 at a distance of 60

$111 \mathrm{~cm}$ from the beginning of the profiles. Data acquisition was carried out in the frequency range $0.8-3$

$112 \mathrm{GHz}$, since the antenna behaved as a dipole. SFR measurements were performed without stop during

113 each series, leading to a huge amount of data. This enabled averaging and stacking of data.

114 This particular air-coupled configuration enabled associating the data with the full-waveform GPR

115 model (Lambot et al. 2004, 2014). This model was used to describe the interaction between

116 electromagnetic waves and the multi-layered medium. The model is based on Green's functions for

117 plane-layered media combined with an intrinsic representation for the antenna through global reflection

118 and transmission functions, to account for antenna effects, including antenna-medium coupling. The

119 considered pavement structure is a three-layered dispersive medium, as shown in Figure 2.

The dispersion in the pavement materials is described by Jonscher's model, which has performed well in describing the frequency-dependence of effective permittivity $\varepsilon_{\mathrm{e}}$ in materials of civil engineering interest (Ihamouten et al. 2011). To simplify the computation and the measurement configuration in the model, the spatial distribution in the near field is assumed to be constant with a single point transceiver,

124 as the antenna height is $4 \mathrm{~cm}$ over the studied medium. This model has been validated numerically and experimentally by Guan et al. (2017).

The generalized model is described in terms of the ratio of reflected to emitted EM waves $S_{11}$. The objective of the inversion process is to reach the best set of model parameters that minimize the difference between analytical radar data $S_{I I}^{\text {mod }}$ (model) and the reference data $S_{l l}{ }^{\text {ref }}$ (experimental data in this study), in the working frequency band. This difference, generally described as a cost function, is

130 called a "residual" hereafter. Since Jonscher's model has been chosen for the multi-layered pavement

131 structure, the model parameters of $\varepsilon_{e}$ (integrated in Green's function) include reference susceptibility $\chi_{e}$,

132 loss exponent $n$, instantaneous dielectric response $\varepsilon_{\infty}$, direct-current conductivity $s \sigma_{d c}$, as seen in Eq. 1,

133 and the thickness $h$ of each layer of the modelled pavement structure. This is given by, 


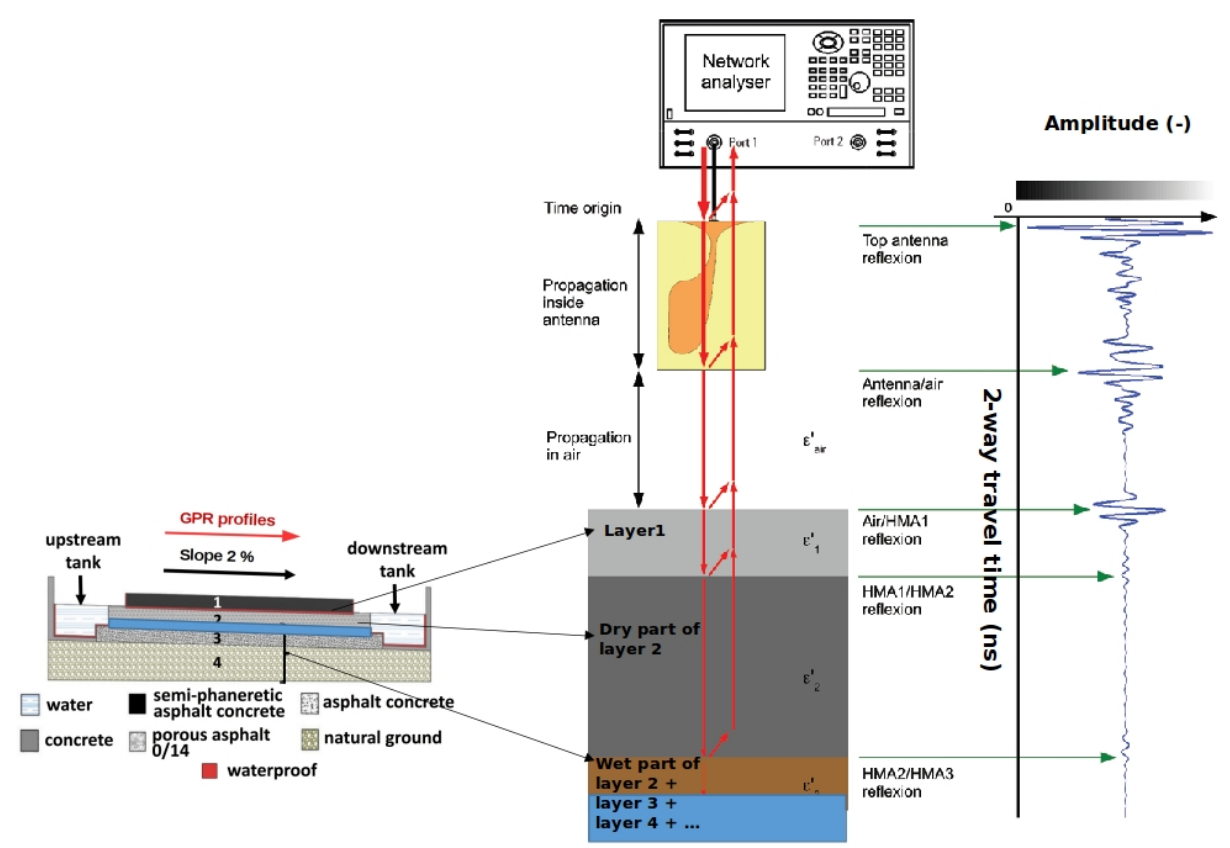

Fig. 2. Fixed configuration of SFR on the pavement test site

$$
\varepsilon_{e}(\omega)=\varepsilon_{0} X_{r}\left(\frac{\omega}{\omega_{r}}\right)^{n-1}\left[1-j \cot \left(\frac{\mathrm{n} \pi}{2}\right)\right]+\varepsilon_{\infty}-j \frac{\sigma_{\mathrm{dc}}}{\omega}
$$

The geometry examined is simplified to 3 layers, leading to a total of 14 model parameters in the analytical model: $\chi_{e}, n, \varepsilon_{\infty}, s \sigma_{d c}$ and $h$ of each layer. The 3 layers are WL, the dry PAL, and a virtual layer composed of the saturated PAL, the BL and the natural ground (infinite thickness).

Due to the fact that the medium below the dry porous asphalt, during the imbibition experiment, is a

141 highly dispersive layer containing water, natural ground, etc., the entire medium below is considered as an equivalent dispersive layer whose dielectric characteristics are constant from one simulated hydric state to another. This hypothesis enables reducing the number of unknowns and making the inversion

144 process more robust. The water penetration depth is then estimated by inversion of SFR data at all time 145 points during the imbibition process.

146 The inversion procedure is, therefore, carried out in two steps. First, in the dry state T0, all layer

147 thicknesses are considered as known and the inversion of the SFR signals determines the 12 dielectric 
parameters of Jonscher's model for the three layers (WL, dry PAL and equivalent layer). The second step of inversion considers the values of all inverted parameters from the first step as fixed and determines only the thickness of the dry porous asphalt layer. This thickness (varying theoretically from

$1518 \mathrm{~cm}$ to $0 \mathrm{~cm}$ ) is inversely proportional to the water front height in layer 2 (varying theoretically from 0

$152 \mathrm{~cm}$ to $8 \mathrm{~cm})$.

\section{Numerical validation of the SFR inversion procedure}

To verify the hypothesis of an equivalent dispersive layer and evaluate its bias, we have carried out a numerical parametric study using a high-frequency simulation software (HFSS) from the ANSYS enterprise. We then compare the FWI results in terms of the residual for a complete and exact configuration made of 5 layers (Fig. 3) and for a 3-layer equivalent configuration. In considering only one medium, the values of $\chi_{e}, n, \varepsilon_{\infty}, s \sigma_{d c}$ and $h$ shown in Fig. 3 for each layer are calculated separately in the first step, using HFSS.

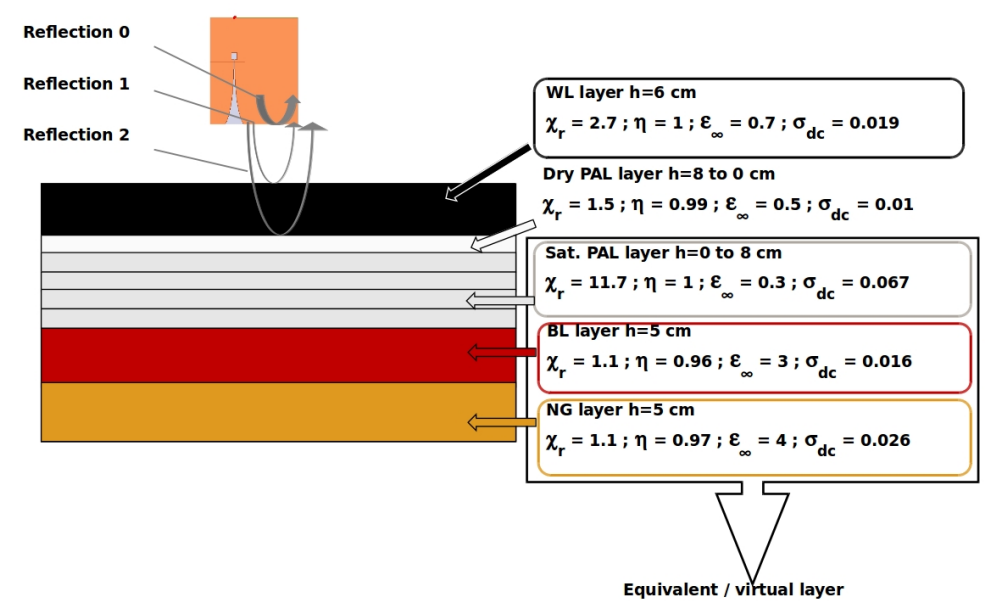

162 Fig. 3. Configuration of the SFR wave propagation numerically simulated using high-frequency simulation software (HFSS) and considering a multi-layer model representing the rise of water in the PAL 

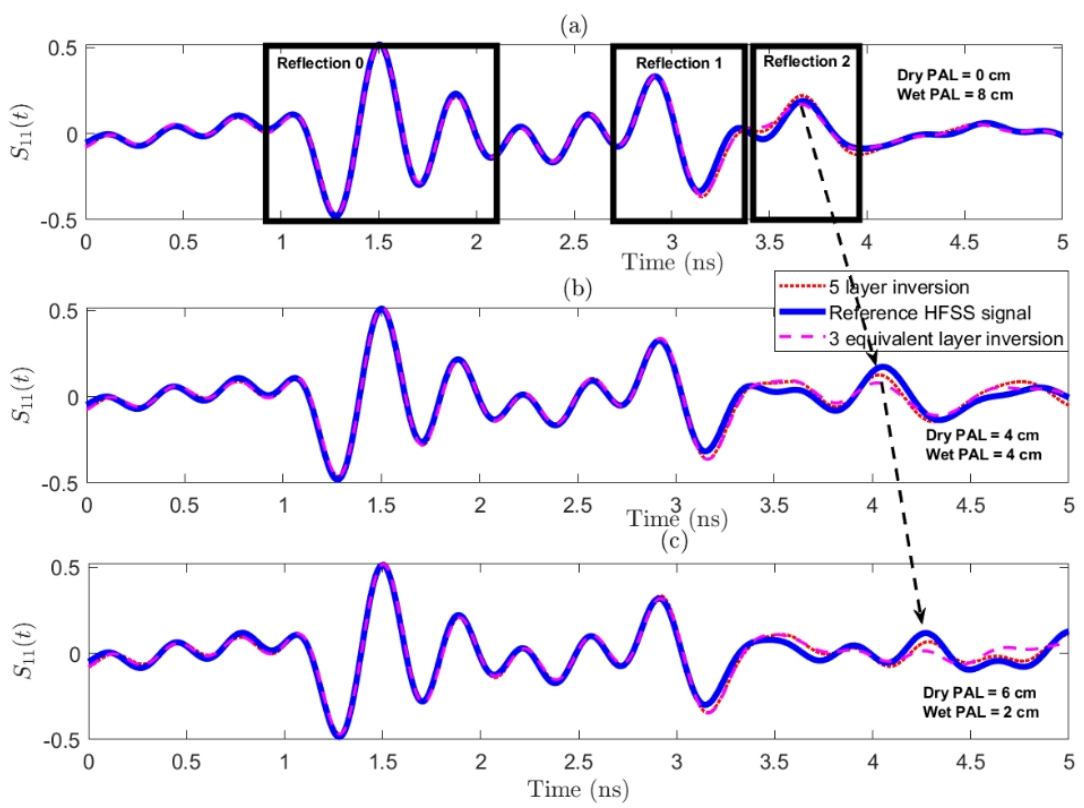

165 Fig. 4. Result of numerical simulation using HFSS (blue line) and FWI using 5-layered (red dotted line) and 3layered (magenta dashed line) models, with the water level located at a) $8 \mathrm{~cm}$, b) $4 \mathrm{~cm}$, and c) $2 \mathrm{~cm}$.

167 Fig. 4 shows an example of numerical results with a temporal representation of the HFSS reference signal (in blue), FWI signals for a 5-layer configuration (in red) and for a 3-layer equivalent configuration (in magenta) for 3 simulated hydric states (with the water front at a) $8 \mathrm{~cm}, \mathrm{~b}$ ) $4 \mathrm{~cm}$, and c) $2 \mathrm{~cm})$. It can be noticed that FWI can perfectly describe the near-field antenna coupling for various models. For any studied configuration, there is a close superposition of signals for the antenna internal reflections (Reflection 0), the reflection from the air/WL interface (Reflection 1) and that from the dry/saturated interface (Reflection 2). However, it can also be seen that the assumption of a 3-layer equivalent medium degrades the inversion results for Reflection 2 and for a relatively thick dry PAL. This is due to the fact that the signals corresponding to the equivalent medium are initially inverted assuming a highly dispersive medium, which considerably attenuates the waves passing through the dry/saturated PAL interface. In contrast, for the case where the dry PAL has a high thickness value (compared to the saturated PAL), the reflections from the lower interfaces (PAL/BL, BL/NG, etc.) are no longer negligible. To evaluate the generality of this bias, we present in Table 1 the FWI residuals for the two configurations examined (5 and 3 layers) for each simulated water state. 
Table 1. Comparison of FWI residual for two configurations using 5-layered and 3 layered models and for each simulated water state

\begin{tabular}{|c|c|c|}
\hline & $\begin{array}{c}\text { FWI residual } \\
\text { 5-layer (complete) configuration [-] }\end{array}$ & $\begin{array}{c}\text { FWI residual } \\
\text { 3-layer (equivalent) configuration [-] }\end{array}$ \\
\hline \multicolumn{3}{|l|}{ Saturated PAL $=8 \mathrm{~cm}$} \\
\hline Dry Pal $=0 \mathrm{~cm}$ & 0.2931 & 0.2855 \\
\hline \multicolumn{3}{|l|}{ Saturated PAL $=6 \mathrm{~cm}$} \\
\hline Dry Pal $=2 \mathrm{~cm}$ & 0.3813 & 0.3871 \\
\hline \multicolumn{3}{|l|}{ Saturated PAL $=4 \mathrm{~cm}$} \\
\hline Dry Pal $=4 \mathrm{~cm}$ & 0.3755 & 0.3678 \\
\hline \multicolumn{3}{|l|}{ Saturated PAL $=2 \mathrm{~cm}$} \\
\hline Dry Pal $=6 \mathrm{~cm}$ & 0.4188 & 0.3681 \\
\hline
\end{tabular}

The results presented in Table 1 confirm those in Fig. 4. The further we move away from the most dispersive state, the higher is the residual. To evaluate the impact of a change from a residual of 0.4188 (5-layer inversion) to a residual of 0.3681 (3-layer equivalent inversion) on the estimated water heights, in Fig. 5 we illustrate an example of the variation in residual as a function of estimated water heights (in this example, Saturated PAL $=4 \mathrm{~cm}$ and Dry PAL $=4 \mathrm{~cm}$ are the references).

Other similar calculations (for various saturated PAL thicknesses) show an increase in the residual by

190 the same order when the error exceeds $1 \mathrm{~cm}$. This confirms that the inversion results derived for a 3-

191 layer equivalent configuration, with regard to low levels of residuals, are at the precision levels of a few

192 millimeters (Fig. 5 for the saturated PAL example $=4 \mathrm{~cm}$ ). 

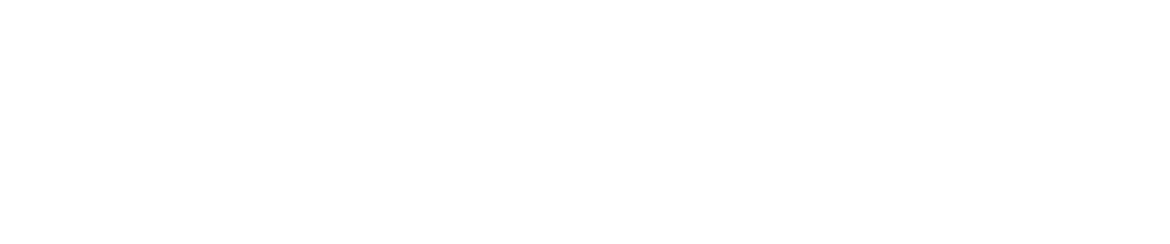

194Fig. 5. Variation of residual with the estimated water height (in this example, saturated PAL $=4 \mathrm{~cm}$ and dry PAL $=4 \mathrm{~cm}$ are the references)

\section{IMPULSE RADAR: RESULTS AND DISCUSSION}

\section{Imbibition campaign}

Transverse B-scans, confirmed by longitudinal GPR profiles made close to the tanks, show that lines

2010 and 8 remained just outside the test site and line 1 was affected by a border. So only lines 2 to 7 were 202 processed during this campaign. Fig. 6a presents a representative raw B-scan during the water imbibition process (series T3, at 16 minutes of imbibition), showing the water front between about 1.6 and $2.2 \mathrm{~m}$, moving towards the right over time. As mentioned earlier, the original structure is clearly

205 detected, as seen in the right part of Fig. 6a. Moreover, it can be seen that the base layer (BL), above the 206 unbounded material, is laid in two stages (slightly visible interfaces at 3 and 3.4-3.6 ns), along with the 207 presence of an embedded thermal sensor located at the WL/PAL interface at about $1.40 \mathrm{~m}$ from the 208 beginning of the profile. 
210 visible in the saturated porous asphalt layer. This level of scattering requires an event picking process

211 which utilizes the main reflection amplitudes: the WL/PAL interface is picked considering the black

212 polarity while the water interface is picked on the white polarity, given the opposite permittivity

213 contrasts.

214 The location of the water front becomes ambiguous when the water reaches WL. This interaction 215 merging the two elementary echoes induces time shifts and amplitude modification that affect the 216 picking of both interfaces. We cannot, therefore, ensure a good correspondence between the picked 217 horizon and the WL/PAL interface. This effect is visible in the left in Fig. 6b,- the porous asphalt layer

218 being saturated. Considering the PAL to be fully saturated, the white-polarity picking of the water front

219 should replace the WL/PAL interface picking at T0 before imbibition. Moreover, WL/PAL picking 220 shown in the left side in Fig. $6 \mathrm{~b}$ is not representative of the interface location. This step is executed only 221 to evaluate the level of disturbance in the polarity of the reflection event during monitoring.

a)

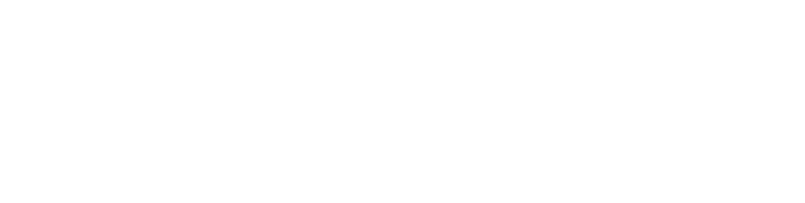

b)

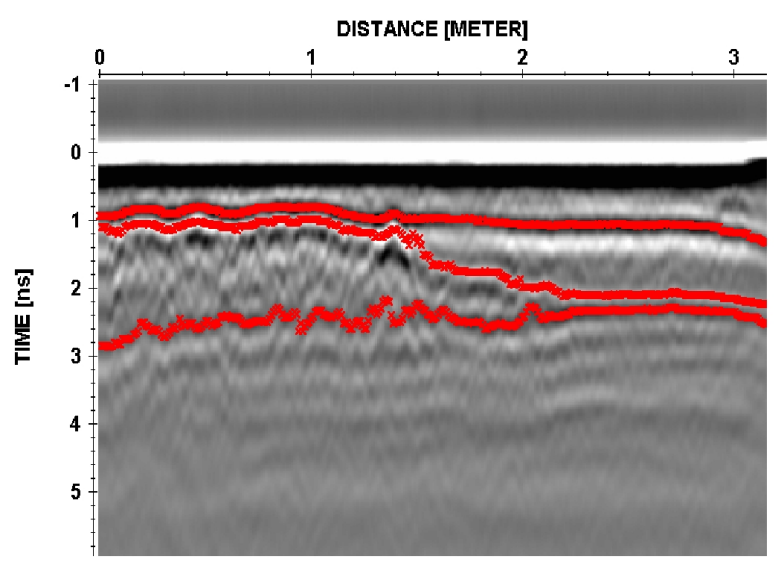

222 Fig. 6. a) Raw impulse GPR B-scan, performed on Line 3 at series T3 (16 minutes of imbibition); b) Same Bscan with horizons picked. 
The following two picked interfaces (WL/PAL and water front) present a nearly half-period decay as visible in Fig. 6b, thus suggesting a very thin layer of dry PAL. Nevertheless, the limited resolution causing overlap of the two echoes creates a problem in picking the correct polarity and the correct location, in the transition zones (before approximately $1.4 \mathrm{~m}$ and after $2.2 \mathrm{~m}$ for the case of series T3 in Fig. 6b).

This phenomenon is similar to the third picked layer, as the first arrival (white polarity) is not visible at the bottom of the saturated part (see on the left in Fig. 6b). Concerning the right part (after $2.2 \mathrm{~m}$ in Fig. 6b), where water front has not yet reached, the visible polarity of the PAL/BL interface suggests an EM property contrast decreasing with depth despite decreasing porosity. This implies some remaining water at the bottom of the PAL, after the purge conducted before the imbibition campaign (not visible by impulse GPR, possibly due to capillary effects masking the water). This hypothesis suggests that additional caution is required in standard GPR survey, when estimating thickness of open porous layers, using time picking .

While picking the black polarity for WL/PAL reflection during the monitoring (Fig. 7a), a time shift can be seen in the left part due to the apparent saturation of the PAL. This effect becomes invisible after $1.4 \mathrm{~m}$. At this specific location, the presence of an embedded thermal sensor, combined with the effect of topography at this interface, probably explain the different behaviour of the flow path on each side.This time shift after first $1.4 \mathrm{~m}$ is confirmed in Fig. $7 \mathrm{~b}$ while picking the water-front reflection (white polarity). Nevertheless, as mentioned above, the water front arrival time should have reached the WL/PAL interface before the imbibition. This time difference (about $0.1 \mathrm{ns)}$ ) suggests an incomplete saturation in this part, although not visible on the profiles. The water flow is visible from series T2 (eleventh minute), going through the structure in less than 40 minutes. Moreover, our results show that a complete saturation of the PAL is not reached in the steady state over the last meter.

Monitoring of the water front is summarized in Fig. 7b, showing also the fluid transfer velocity. The water front reaches the opposite side of the road in about 24 minutes (between T4 and T5). The right 
249 part of the structure is then filled with water. It can be noticed that an air lens, between 1.5 and $3.2 \mathrm{~m}$,

250 remains visible in the steady state (after series T8), as the difference in the picked polarity does not

251 explain the time shift between WL/PAL interface and the water front.

a)

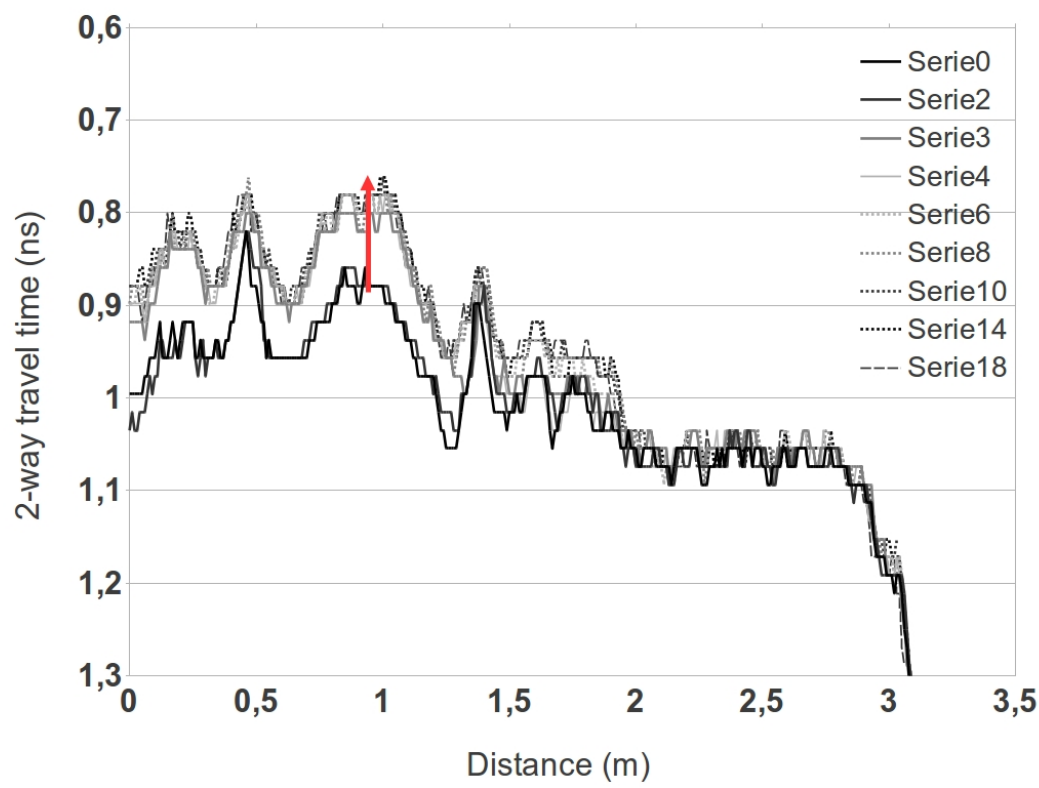

b)

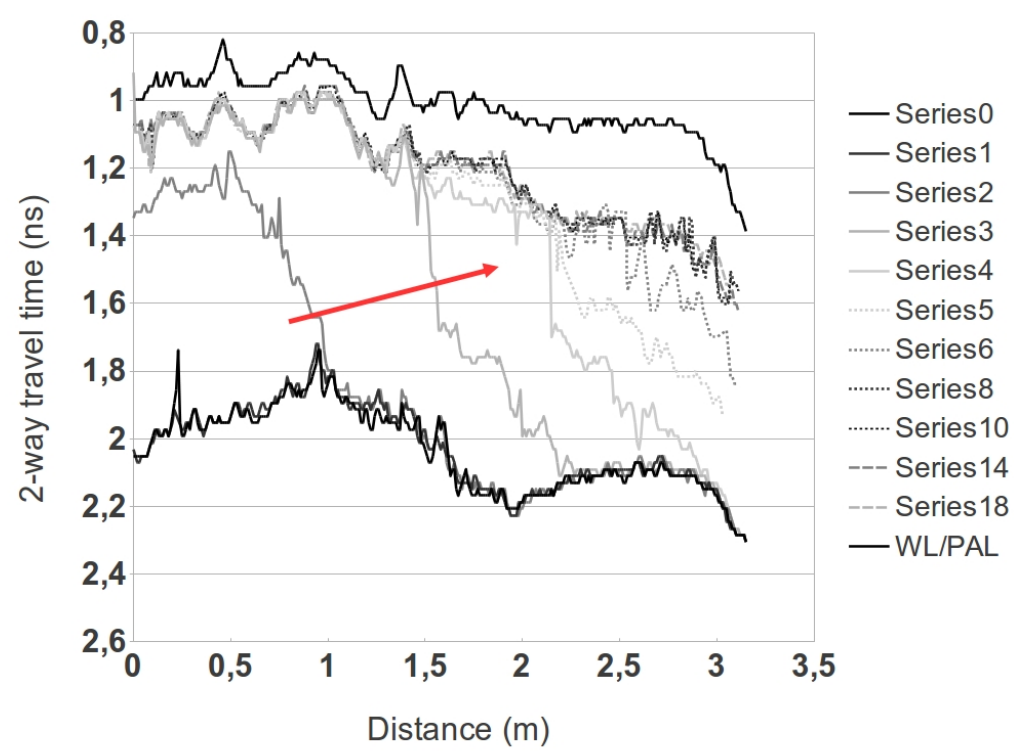

252Fig. 7. Time picking for different series performed on Line 3: a) on the WL/PAL interface, b) for the water front entering PAL. The WL/PAL black curve corresponds to series 0 . 
255 Event picking was performed on every line for the water front and gathered to form a map, showing 256 the variation of travel times due to water flow (T0-Ti). Fig. 8 shows the progress of water flow within 257 the first 8 series, and no more notable evolution after this time (see Fig. 8f representing a nearly steady 258 state). It should be noted that for series $\mathrm{T} 3$ to $\mathrm{T} 8$ on Line 4, it was not possible to detect and pick the 259 water-front reflection all along the profile. As a consequence, two dark areas remain in the maps.

a)

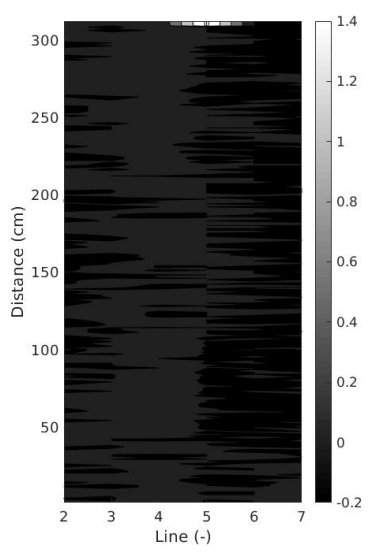

d)

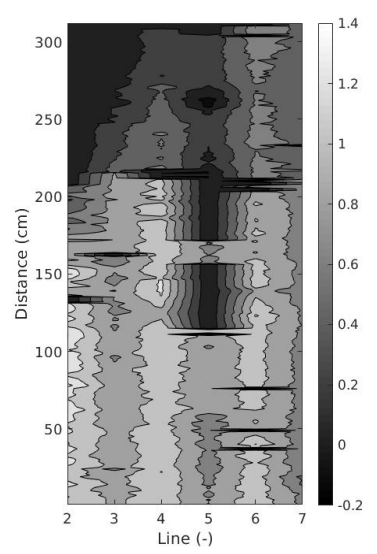

b)

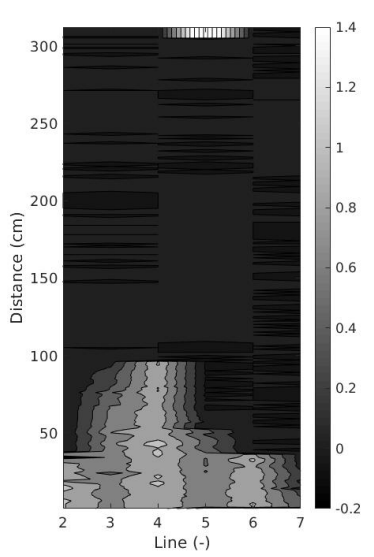

e)

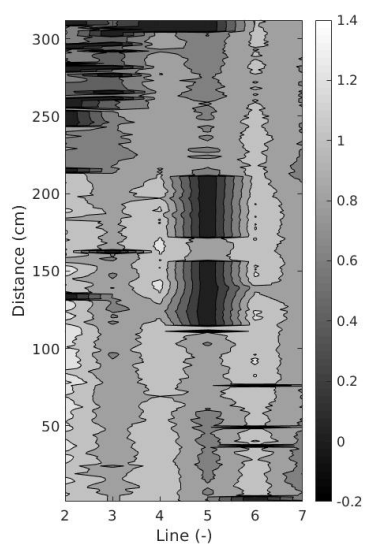

c)

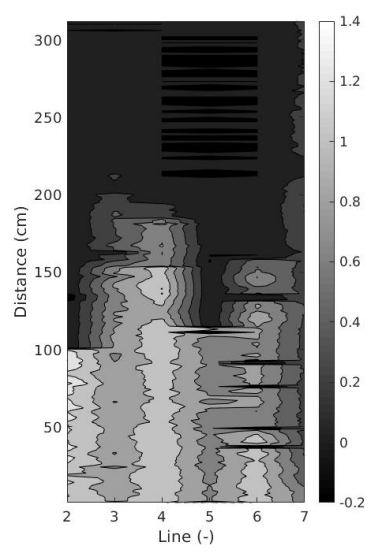

g)

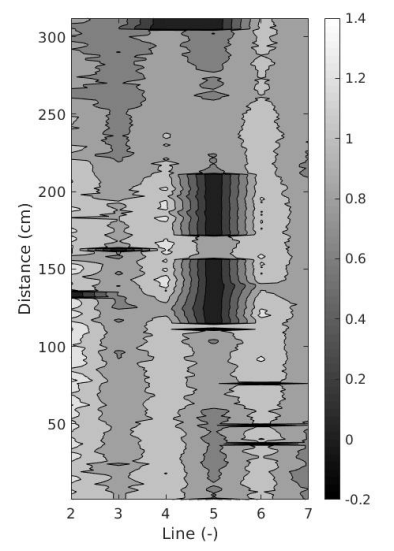

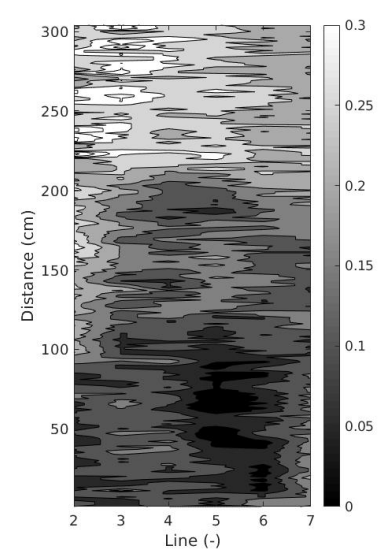

260 Fig. 8. Impulse GPR monitoring showing variations of reflection travel times for the water front (in ns), a) T0261 T1, b) T0-T2, c) T0-T3, d) T0-T4, e) T0-T6, f) T0-T8. g) Picking the time shift between the W/PAL interface (T0) and the water front (T8) in the steady-state flow. 

front in the steady state. It can be seen that the PAL is not fully saturated. This is explained by the fact 265 that the edge of the downstream tank is located at the bottom of the PAL. As a consequence, the water 266 flow is not sufficient to counterbalance the water leakage in the downstream area.

267 Impulse GPR monitoring does not detect the clear air lens trapped on top of the PAL. These results 268 show the possibility of monitoring the water flow along a transverse slope, as well as along a slightly 269 longitudinal slope.

272 During the purge and water leakage, capillary effects did not induce any sharp water front. 273 Moreover, the high EM contrasts in the PAL induced visible scattering in the B-scans (Fig. 9a) and, 274 therefore, no clear EM interface to be detected by impulse GPR. As seen in the T'16 B-scans of Line 3 275 (Fig. 9b), the visible water table was detectable after more than 1.5 hour.

a)

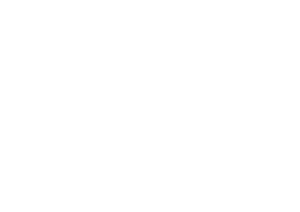

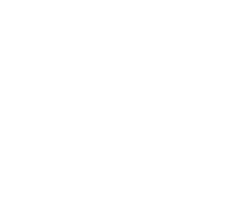

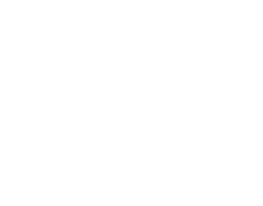

b)
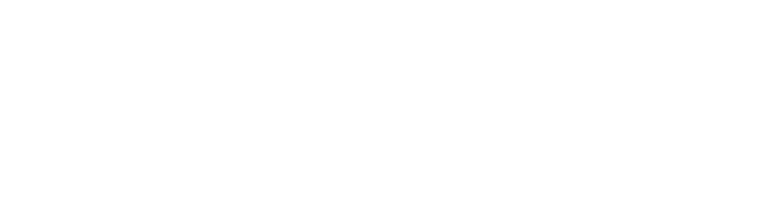

276 Fig. 9. GPR B-scans performed on Line 3 during the purge: a) at $\mathrm{T}^{\prime} 0$ (series 25), b) at $\mathrm{T}^{\prime} 16$ (series $\left.41-3 \mathrm{~h}\right)$. 
The effect of water leakage on impulse GPR measurements was visible from the start, allowing time picking for the bottom interface of the PAL. Average permittivity, decreasing with a decrease in the average water content, increased the GPR wave velocities and hence reduced the arrival times.

The results are summarized in Fig. 10, showing shifts in arrival time (2-way traveltime) from upstream (left part of the curves) to downstream (right part). This effect is visible around 1 hour 50 minutes (series 33) after the start of the purge. Dispersion in the picked travel times can be noted due to the high level of scattering in the PAL (see Fig. 9a) due to high EM contrasts.

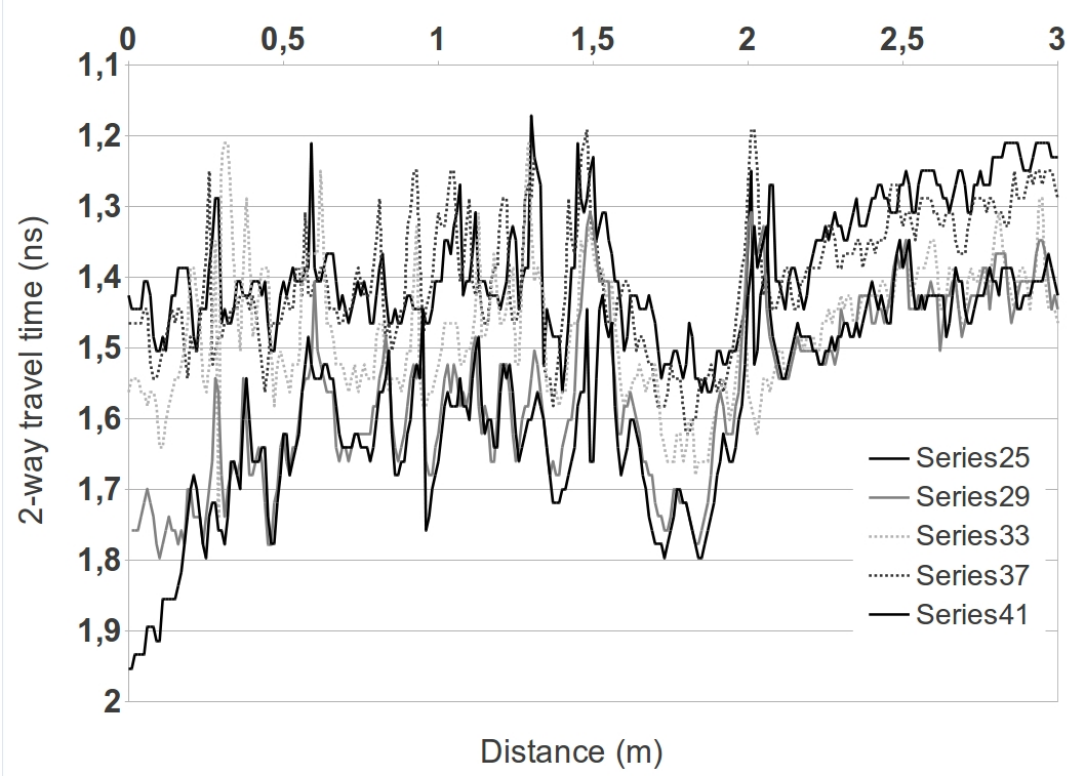

Fig. 10. Time picking for the PAL/BL interface for different series performed on Line 3.

\section{SFR RESULTS AND DISCUSSION}

The SFR inversion process is detailed for the initial test time (T0) by way of an example. The 289 objective function gives a minimal value of 0.052 , which indicates that the match is good in the 290 frequency domain. The correspondence between analytical (model) and experimental GPR data is 291 shown in Fig. 11. 
Note that the small differences are due to the assumption of the so called "equivalent layer". It

293 should also be mentioned that the reflections in the time domain (see Fig. 11c) are hard to distinguish,

294 as they are very similar. In this case, it is not possible to use the usual amplitude inversion in the time

295 domain, but full waveform inversion in the frequency domain remains a powerful tool for such a case.

296 This inversion returns a global minimum, as shown in Fig. 12. The inversion results for the

297 experimental radar data, which shows the inverted Jonscher's model parameters of the pavement layers,

298 are presented in Table 2 and in Fig. 13 in terms of complex permittivities of each layer.

(a)

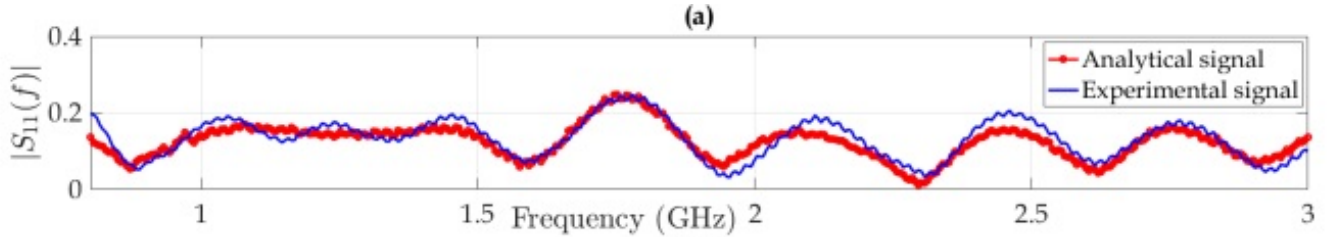

(b)

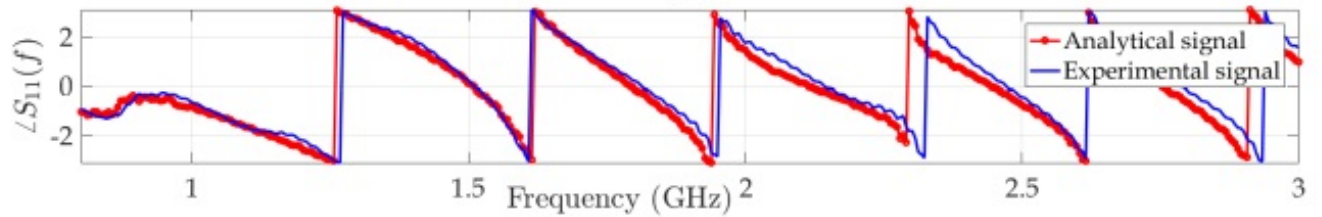

(c)

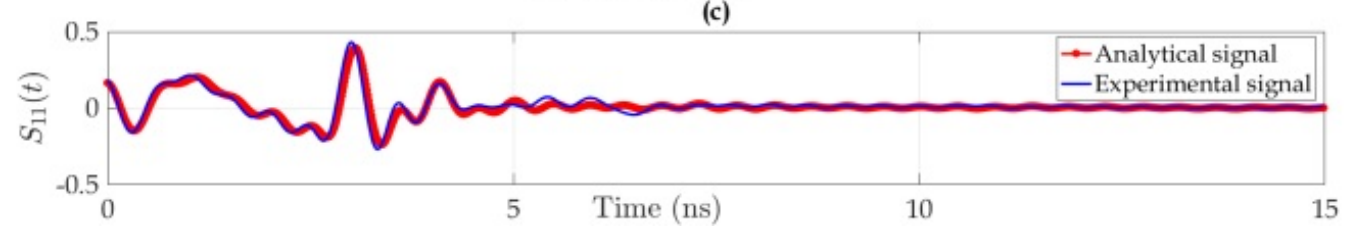

299

300Fig. 11. Comparison between analytical and experimental signals, $S_{11}{ }^{\text {mod }}\left(f_{i}, m\right)$ and $S_{11}{ }^{\text {ref }}\left(f_{i}\right)$, at the end of inversion. 
Best: 0.052055 Mean: 0.063314

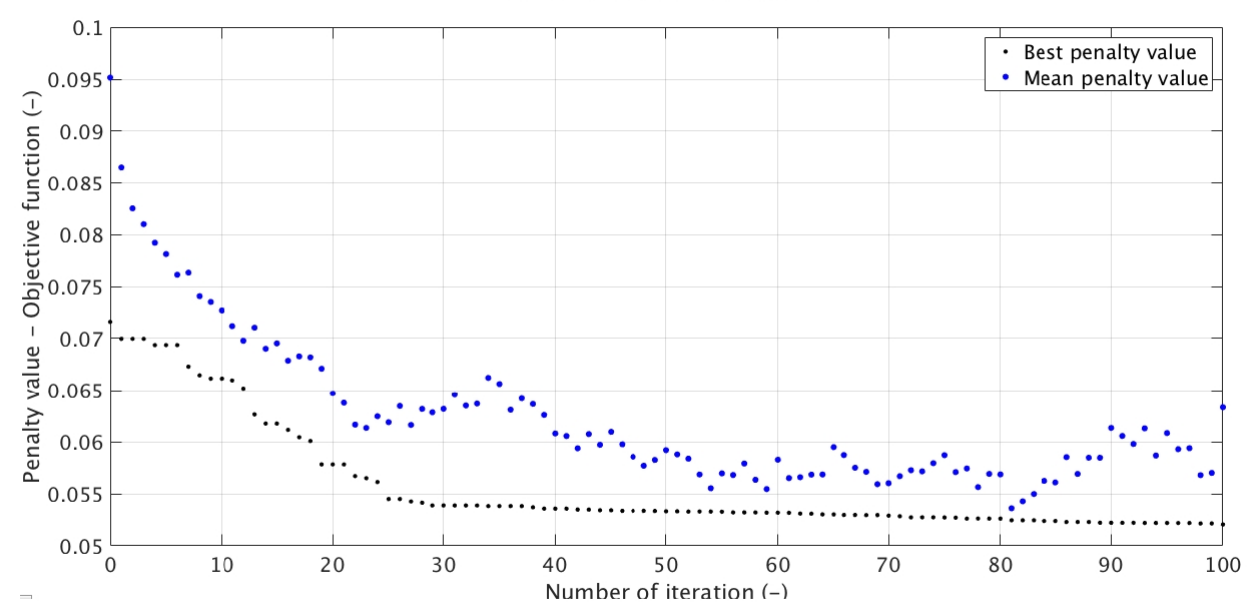

301

Fig. 12. Evolution of the objective function as a function of iteration number.

(a)

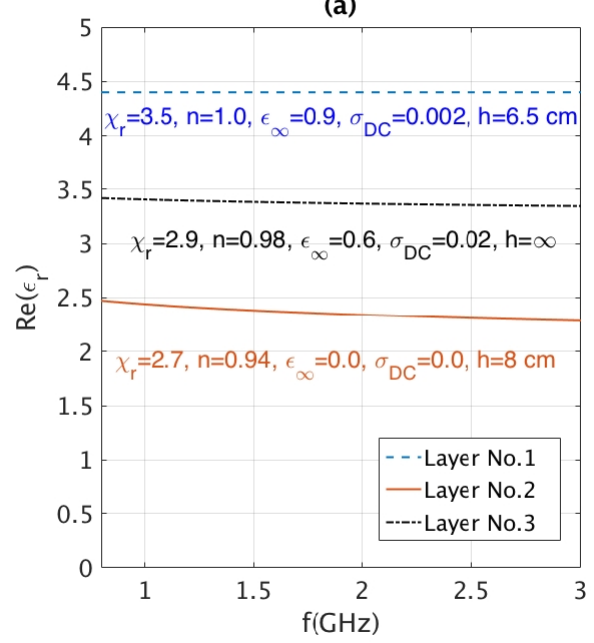

(b)

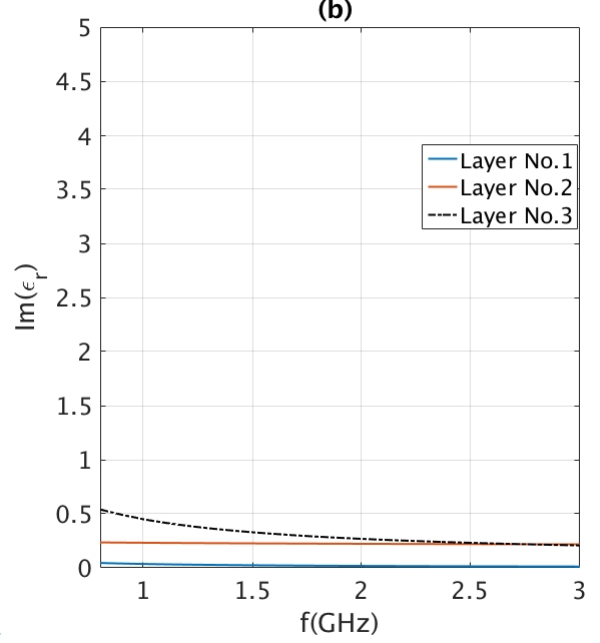

Fig. 13. Inverted effective permittivities for the three layers considered.

306 It should be noted that these results are obtained in the initial dry state T0, where the thicknesses of

307 each layer are well-known. These inverted parameters (Table 2) will be considered in the rest of the

308 experimental/inversion process as invariables (inversion input), and the inversion output will then be

309 limited to the thickness of the dry porous asphalt layer (dry part of layer 2). 


\begin{tabular}{|c|c|c|c|c|c|}
\hline Layer & $\boldsymbol{X} r$ & $\boldsymbol{n}$ & $\boldsymbol{\varepsilon}_{\infty}$ & $\boldsymbol{s}_{d c}$ & $\begin{array}{c}\boldsymbol{h}(\mathbf{k n o w n}) \\
{[\mathbf{c m}]}\end{array}$ \\
\hline Layer 1 & 3.5 & 1.0 & 0.9 & 0.002 & 6.5 \\
\hline Layer 2 (dry PAL) & 2.7 & 0.94 & 0.0 & 0.000 & 8 \\
\hline Virtual equivalent layer 3 & 2.9 & 0.98 & 0.6 & 0.020 & - \\
\hline
\end{tabular}

312 Direct measurement (not shown in the present paper) at a fixed point shows the penetration of the 313 water into the material. It allowed us to follow (as with the impulse radar data) the intrusion of the 314 water front into layer 2. However, temporal time picking gives an indication of the evolution of the 315 water front but not of its exact position. We, therefore, carried out inversions of the SFR signals at times $316 \mathrm{~T} 0, \mathrm{~T} 1, \mathrm{~T} 3, \mathrm{~T} 4, \mathrm{~T} 5, \mathrm{~T} 6, \mathrm{~T} 12, \mathrm{~T} 17$ and $\mathrm{T} 21(\mathrm{~T} 0+7$ minutes, $\mathrm{T} 0+20$ minutes, $\mathrm{T} 0+27$ minutes, $\mathrm{T} 0+34$ 317 minutes, $\mathrm{T} 0+40$ minutes, $\mathrm{T} 0+70$ minutes, $\mathrm{T} 0+98$ minutes and $\mathrm{T} 0+130$ minutes, respectively) to 318 estimate the thickness of the dry PAL (dry part of layer 2). The results showing the inverted thicknesses 319 as a function of the imbibition time are plotted in Fig. 14. It can be seen that the water penetration front 320 does not reach the interface between layers 1 and 2 . In other words, water only penetrates into the 321 drainage layer up to a maximum height of $6 \mathrm{~cm}$ instead of the target $8 \mathrm{~cm}$. 


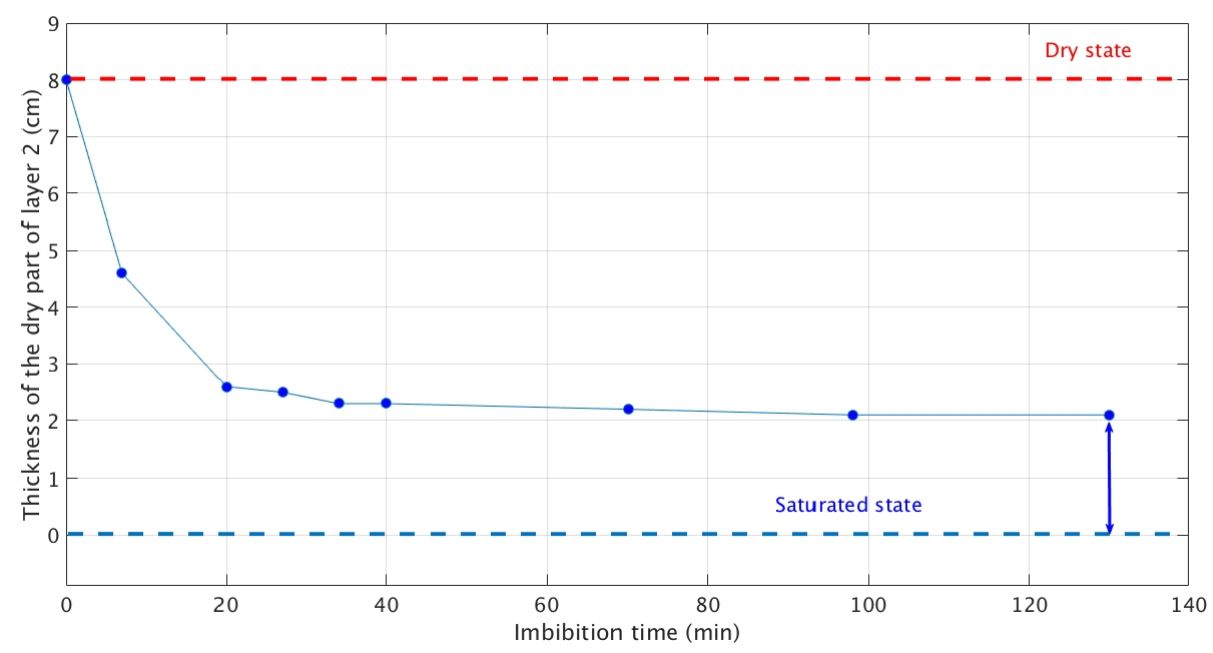

Fig. 14. Evolution of the estimated dry thickness of the PAL during monitoring.

These results may, however, be biased due to the different water gradients at the location of the pavement. The slope at this measurement point is in two directions. This, incidentally, explains the results of the impulse GPR measurements for Line 6. Another explanation for this time shift pertains to the initial hydric state of the pavement structure. Before $\mathrm{T} 0$ of the experiment, a water purge had been performed, with some humid gradients possibly remaining due to the capillary effects. This could lead to a biased estimation of the Jonscher's parameters in the initial state, for which the associated initial value of $8 \mathrm{~cm}$ for the thickness of layer 2 is not realistic. EM properties of the third equivalent layer (fixed for every inversion). The water flow may induce a non-negligible bias in the fixed values defining the equivalent layer 3 and then cause a bias in the estimation of the dry thickness. Even though the accuracy of the estimated thickness values may be debatable, the monitoring experiment has shown the efficiency of NDT using GPR. 
The monitoring of water imbibition in a porous pavement structure was performed using impulse radar and SFR techniques. Impulse GPR data allowed us to follow the progress of the water front through picking the travel times. In parallel, static SFR measurements were continuously performed and the data were inverted using an FWI scheme in the frequency domain. The impulse GPR results allowed us to follow the water front and showed different behaviour for the water transfer in the PAL, which could be explained by the vertical topology of the upper water-tight interface. This monitoring campaign illustrated that, when estimating thickness of open porous layers using time picking of reflection events. one should take- sufficient caution.

For the SFR measurements, a numerical parametric study was performed. This was the first step to validate the hypothesis of a virtual equivalent layer. This step defined the saturated PAL, the BL and the NG, thereby reducing the number of unknowns in the inversion and facilitating the convergence, while limiting the number of local minima. The parameter studied here is the residual. The numerical results showed that the bias caused by the equivalent thickness assumption induces an error which is much less than one $\mathrm{cm}$.

The SFR and the impulse GPR techniques offer similar results, but the SFR provides quantitative information on the height of the water front inside the PAL. The two approaches have been tested and adapted for monitoring water transfer in porous pavement structures.

\section{REFERENCES}

Pan P., Wu S., Xiao Y. and Liu G. 2015. A review on hydronic asphalt pavement for energy harvesting and snow melting. Renewable and Sustainable Energy Reviews 48, 624-634.

Andriopoulou S. 2012. A Review on Energy Harvesting From Roads. Master thesis, KTH, School of Architecture and the Built Environment, p.39. 
Bobes-Jesus V., Pascual-Muñoz P., Castro-Fresno D. and Rodriguez-Hernandez J. 2013. Asphalt solar collectors: a literature review. Applied Energy 102, 962-970.

Asfour S., Bernardin F., Toussain E. and Piau J.M. 2016. Hydrothermal modeling of porous pavement for its surface de-freezing. Applied Thermal Engineering 107, 493-500.

Lai W.L., Dérobert X., Annan A.P. 2017. A review of Ground Penetrating Radar Application in Civil Engineering: a 30-year journey from Locating, Testing and Evaluation to Imaging and Diagnosis. Non Destructive Testing \& Evaluation International 96, 58-78.

Saarenketo T. and, Scullion T. 2000. Road evaluation with ground penetrating radar. Journal of Applied Geophysics 43, 119-139.

Loizos A. and Plati C. 2007. Accuracy of pavement thicknesses estimation using different ground penetrating radar analysis approaches. Non Destructive Testing \& Evaluation International 40, 147 157.

Dérobert X., FauchardC., Côte Ph., Le Brusq E., Guillanton E., Dauvignac J.Y. and, Pichot Ch. 2001. Step frequency radar applied on thin road layers. Journal of Applied Geophysics 47, 317-325.

Guan B., Ihamouten A., Dérobert X., Lambot S. and Villain G. 2017. Near-field full-waveform inversion of radar waves to monitor water front in limestone. Journal of Selected Topics in Applied Earth Observations and Remote Sensing 99, 1-9.

Lambot S., Slob E., Van den Bosch I., Stockbroeckx B. and Vanclooster M. 2004. Modeling of ground-penetrating radar for accurate characterization of subsurface electric properties. IEEE Transactions on Geoscience and Remote Sensing 42, 2555-2568.

Lambot S. and Andre F. 2014. Full-wave modeling of near-field radar data for planar layered media reconstruction. IEEE Transactions on Geoscience and Remote Sensing 52, 2295-2303.

Ihamouten A., Chahine K., Baltazart V., Villain G. and Dérobert X. 2011. On variants of the frequency power law for the electromagnetic characterization of hydraulic concrete", IEEE Transactions on $I$ 